\title{
Design of composite right/left-handed transmission line for phase shifter of multi-band base station antenna
}

\author{
Naobumi Michishita ${ }^{1, *}$, Yoshihide Yamada ${ }^{2}$, and Keizo Cho ${ }^{3}$ \\ ${ }^{1}$ Department of Electrical and Electronic Engineering, National Defense Academy, Yokosuka 239-8686, Japan \\ 2 Department of Electronic Systems Engineering, Malaysia-Japan International Institute of Technology, Universiti Teknologi \\ Malaysia, Jalan Sultan Yahya Petra, 54100 Kuala Lumpur, Malaysia \\ ${ }^{3}$ Department of Information and Communication Systems Engineering, Chiba Institute of Technology, Narashino 275-0016, \\ Japan
}

Received: 3 September 2018 / Accepted: 17 November 2018

\begin{abstract}
In multi-band base station antennas, interference toward neighboring cells at low operating frequency in-creases since tilting angle is frequency-independent and beamwidth in the vertical plane increases as de-creasing the operating frequency. In this paper, the realization of the different phase shifts at each frequency is proposed by using the dispersion relation of the composite right/left-handed transmission line. The feasi-bility of the phase shift is clarified by using equivalent circuit model of the unit cell. Next, the dispersion and Bloch impedance of the actual configuration of the unit cell are designed. The inverse phase shift at each frequency is verified experimentally.
\end{abstract}

\section{Introduction}

In mobile communication systems, cellular configuration is commonly used [1]. In addition, due to the recent explosive increase of mobile communication traffic, multiple frequency bands have been assigned for mobile communication systems. Therefore, base station antennas for the mobile communication systems require to operate in multiple frequency bands.

The mobile base station antenna is generally composed of vertically aligned sub-arrays, and each sub-array comprises vertically aligned several elements [2]. In the cellular system, the main beam of the base station antennas in the vertical plane is down-tilted to suppress the interference toward neighboring cells [3]. The beam tilt is achieved by changing the phases of sub-arrays by using the phase shifter [4]. The phase shifter comprises movable dielectric plates [5] or metal strips [6], and the phase difference is achieved by changing the electrical or physical line length with shifting the dielectric plates [5] or the metal strips [6].

In the multi-band base station antennas, the electromagnetic wave propagating along the transmission lines in the phase shifter is usually independent to the frequency. The excitation phase to tilt the beam is given by the

\footnotetext{
* e-mail: naobumi@nda.ac.jp
}

multiplication of the wave number $k$ and the length $d_{p}$ from the subarray to the wave front. When the transmission lines corresponding to the length $d_{p}$ are added in the phase shifter, since electromagnetic wave propagates along the transmission lines independently to the frequency, the tilt angles become identical over the sharing frequency bands in the multi-band base station antenna. However, since the excitation phase depends on the wave number $k$, the excitation phase of each subarray becomes frequencydependent properties. On the other hand, the electrical array aperture of the multi-band antenna decreases according to decreasing the frequency because the physical array aperture is the same. Therefore, since the beamwidth of the base station antenna increases as decreasing the frequency, the interference toward neighboring cells in lower frequency band increases. The interference can be suppressed if the tilt angle increases in low frequency. This requires non-linear phase shift depending on the frequency in the phase shifter while the tilt angle is frequencyindependent when using a conventional phase shifter. The dispersive transmission lines are suitable for the required function.

Recently, composite right/left-handed (CRLH) transmission line has received much attention because CRLH transmission line can achieve unnatural propagation phenomena by appropriately designing its configuration such as backward propagation [7]. By using backward propagation property of the CRLH transmission line, 
the phase shifters composed of coplanar waveguide with lumped element are compact in size and a linear phase response [8]. The linearly and broadband tunable performances can be achieved by using varactor diodes $[9,10]$. The tunable negative index materials are also composed of a periodic copper wire sandwiched between yttrium iron garnet films [11]. Another important aspect in the CRLH transmission line is the phase dispersion characteristics of CRLH transmission line. This property has been applied to frequency beam scanning leaky wave antennas $[12,13]$. Since the passing phase of the CRLH transmission line can be varied from backward to forward by scanning the frequency, wide beam steering can be achieved by using CRLH transmission line.

We have proposed to introduce the CRLH transmission line to the phase shifter of $1.5 / 2 \mathrm{GHz}$ dual-band base station antenna to use the phase dispersion characteristic [14]. By applying the CRLH transmission line to the phase shifter, the tilt angle can be increased in low frequency band. Since base station antennas are placed at various locations, the tilt angle has to be adjusted to suppress the interference toward the neighboring cells in each location. However, unlike the conventional beam steering leaky wave antennas using the CRLH transmission line, frequency cannot be changed to adjust the beam direction in communication systems. In our proposed phase shifter, mechanically movable patches are placed above the transmission line then metal insulator metal (MIM) capacitors are configured. The configuration has achieved to vary the phase dispersion curve.

This paper is the extension of [14] and [15] presented by authors. In this paper, the required phase shift for the proposed phase shifter is first investigated by using a typical base station antenna example. Reference [14] shows the configuration of the proposed phase shifter and simulation results of the proposed CRLH transmission line. In this paper, the requirements to design the unit cell of the CRLH transmission line are studied by using $\mathrm{T}$ shape 2 port network and circuit parameters for the unit cell are derived. Then, the circuit parameters of the realized unit cell structure presented in [14] are derived and the influence of the structural parameters on the circuit parameters is investigated. After that, the characteristics of a cascade structure of the designed unit cells are evaluated by the measurement and simulation. This measured and simulated results are based on [15]. The comparison between the results and 2-port network analysis is also discussed and the feasibility of the proposed phase shifter is evaluated.

The organization of the paper is as follows. In Section 2, the configuration of conventional base station antenna is first shown and required phase shift for the proposed phase shifter is presented. Then, the 2 port network analysis of the unit cell is described in Section 3. The comparison of the circuit parameters between the 2 port network and the realized unit cell is discussed in Section 4. The experimental and simulation results of 5-cell proposed CRLH transmission line are described in Section 5. Section 6 describes the conclusion.

\section{Requirement of phase shift in base station antenna}

\subsection{Conventional base station antenna configuration}

Figure 1 shows a typical mobile base station antenna configuration. We assume that the base station antenna operates from $1.5 \mathrm{GHz}$ band to $2 \mathrm{GHz}$ band and it is composed of 4 sub-arrays. Each sub-array is composed of equi-spaced 4 planer dipole elements. The beam tilt can be achieved by equalizing the phases among the elements. The phase differences among the elements correspond to the distances normal to the wave front.

The feed network in each sub-array is designed to achieve the beam tilt angle of $\theta_{T}$ by changing the lengths of the transmission lines as shown in Figure 1a. The tilt angle of each sub-array is generally designed at the center of the variable tilt angle range. Here, we select the element spacing in the sub-array to be 0.65 wavelengths at $2 \mathrm{GHz}$, and the tilt angle $\theta_{T}=4.5^{\circ}$ as an example [16].

In the conventional base station antenna, each subarray is excited through a variable phase shifter and cables as shown in Figure 1b. The length of the cables are all set to be identical and phase delays required for the lowerinstalled sub-arrays to achieve beam tilt are given by variable phase shifters. In an example of the conventional phase shifter configuration [17], the radial strip line is electromagnetically coupled to the arc strip line through the slot. The pass length of the phase shifter can be varied by changing the angle $\varphi$ of the radial strip line. Since the phase velocities in the air or in the strip lines are the same in all frequencies, the tilt angles are independent to the frequencies.

\subsection{Proposed phase shifter and required phase shift}

Figure 2 shows the directivity difference at the above base station antenna between 1.5 and $2 \mathrm{GHz}$ at the vertical angle $\theta$, when the tilt angle $\theta_{T}=4.5^{\circ}$. As shown in Figure 2 , the directivity difference increases as decreasing $\theta$ from $4.5^{\circ}$. The tilt angle $\theta_{T}$ is usually chosen toward the cell edge direction. When assuming that the base station antenna is located at the height of $40 \mathrm{~m}$, the tilt angle $\theta$ of $4.5^{\circ}$ corresponds to the cell radius of around $500 \mathrm{~m}$. The vertical angles $\theta$ of $2^{\circ}$ and $1^{\circ}$ correspond to the distance of about $1150 \mathrm{~m}$ and $2300 \mathrm{~m}$ from the base station, which are in the next cell and next to the next cell, respectively.

To suppress the interference toward the neighboring cells, we have proposed to give a deeper beam tilt in lower frequency by adding frequency dispersive transmission lines composed of CRLH transmission lines to the conventional phase shifter. Figure 3 shows the configuration of the proposed phase shifter. The required additive phase shift in the low frequency band is investigated here. To equalize the directivities at a specific angle $\theta$ at 1.5 and $2 \mathrm{GHz}$, the additive phase shift for $1.5 \mathrm{GHz}$ is required. Therefore, directivity patterns with the additive phase shift are calculated at $1.5 \mathrm{GHz}$ iteratively until the 


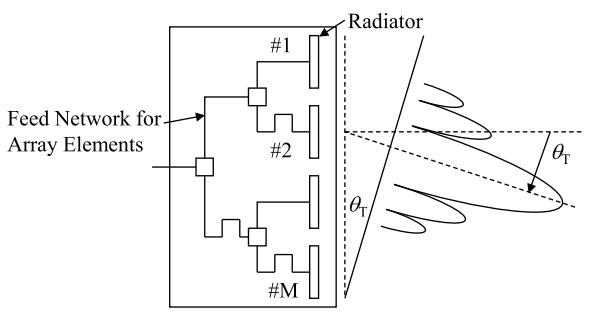

(a)

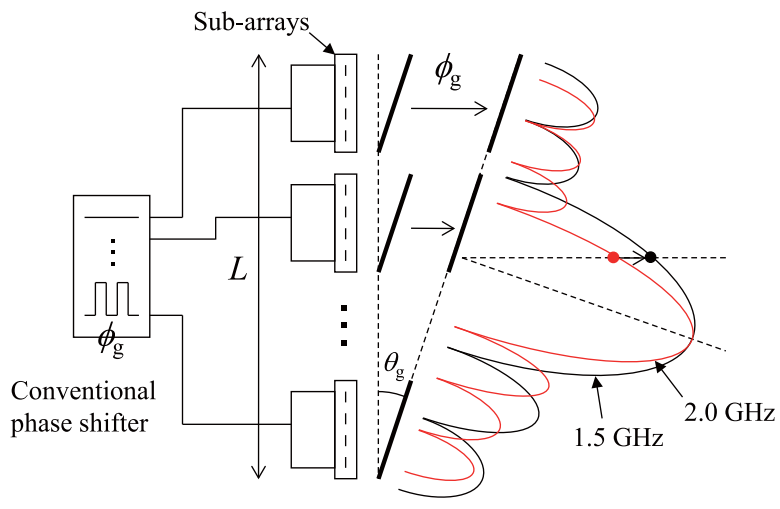

(b)

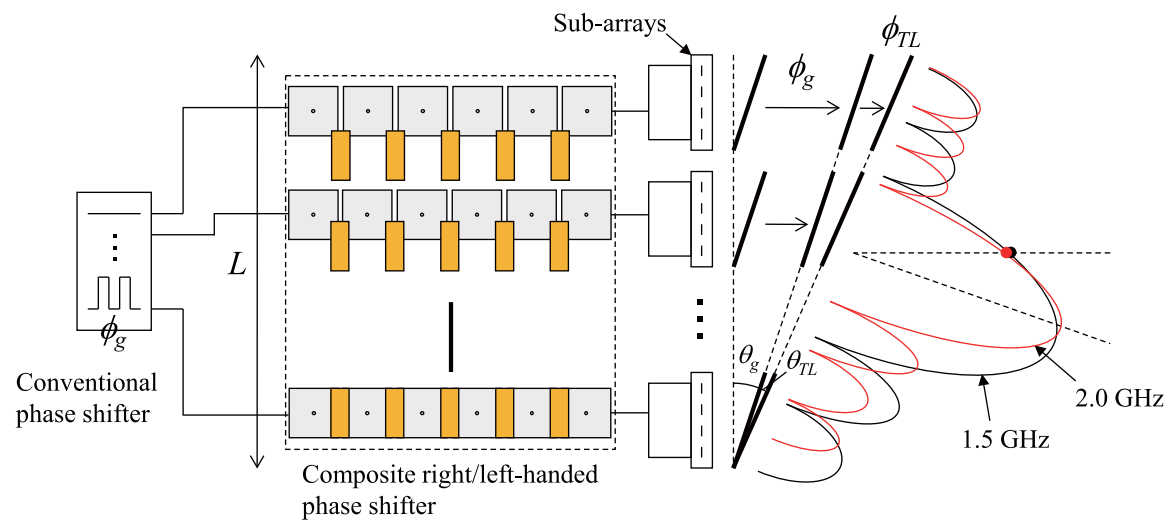

(c)

Fig. 1. Configuration concept of base station antenna. (a) Sub-array composed of 4 array elements. (b) Base station antenna with conventional phase shifter and (c) proposed conposite right/left-handed phase shifter.

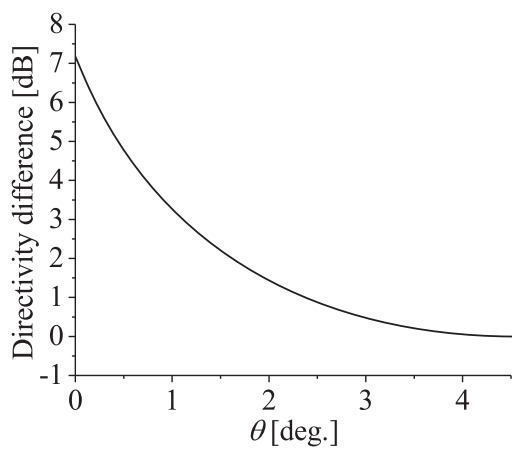

Fig. 2. Directivity difference between 1.5 and $2 \mathrm{GHz}$.

directivities at the angle $\theta$ are equalized at both the frequencies. Figure 4 shows the required maximum phase shift for $1.5 \mathrm{GHz}$ to equalize the directivities toward the vertical angle $\theta$. When equalizing the directivities toward the vertical angles $\theta$ of $2^{\circ}$ and $1^{\circ}$, the beam tilt angle of $1.5 \mathrm{GHz}$ must be increased by $5.38^{\circ}$ and $5.73^{\circ}$. In this case, the maximum of $31.9^{\circ}$ and $44.7^{\circ}$ phase shift are additionally required at $1.5 \mathrm{GHz}$ from the phase at the tilt angle of $4.5^{\circ}$, respectively.

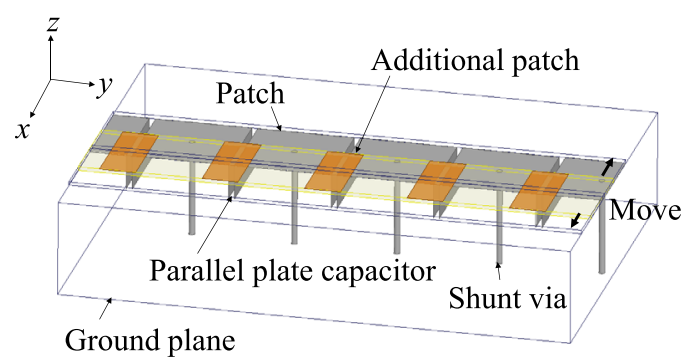

Fig. 3. Configuration of proposed phase shifter.

\section{Equivalent circuit design of unit cell}

This section describes the electrical design requirements for the unit cell of the CRLH transmission line. The equivalent circuit for the ideal CRLH transmission line can be shown as Figure 5. $L_{R}$ and $C_{R}$ are the series inductance and the shunt capacitance which exist in the equivalent circuit of conventional microstrip line. The series capacitance $C_{L}$ is provided by mainly the gap between patches. The shunt inductance $L_{L}$ is provided by mainly the via. 


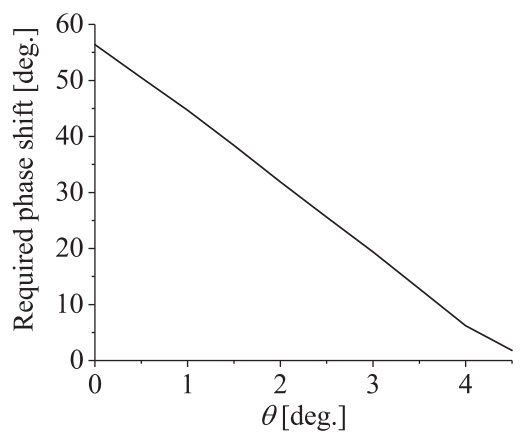

Fig. 4. Required maximum phase shift for $1.5 \mathrm{GHz}$ to equalize the directivities toward vertical angle $\theta$.

Since the proposed phase shifter operates in wide frequency range as from 1.5 to $2 \mathrm{GHz}$ bands, the balanced conditions should be satisfied for achieving the stable Bloch impedance characteristics. By using the circuit parameters, the series and shunt resonant frequencies are expressed as

$$
\begin{aligned}
& f_{\mathrm{se}}=\frac{1}{2 \pi \sqrt{L_{R} C_{L}}}, \\
& f_{\mathrm{sh}}=\frac{1}{2 \pi \sqrt{L_{L} C_{R}}} .
\end{aligned}
$$

In addition, the $f_{\text {se }}$ and $f_{\text {sh }}$ need to be larger than the frequency of $2 \mathrm{GHz}$ band because the operational frequency should not be in the bandgap region. Therefore, the first condition of the design procedure is the balanced case of $f_{\mathrm{se}}=f_{\mathrm{sh}}$, and those should be larger than the $2 \mathrm{GHz}$ bands, which is more than around $2.2 \mathrm{GHz}$.

In the balanced case, the cutoff frequency can be expressed to take the simpler form

$$
f_{c L}=\frac{1}{2 \pi \sqrt{C_{R} L_{R}}}\left|1-\sqrt{1+\sqrt{\frac{C_{R} L_{R}}{C_{L} L_{L}}}}\right| .
$$

The lowest frequency band, which is $1.5 \mathrm{GHz}$ band here, should be above the cutoff region. Therefore, the second condition is that $f_{c L}$ is lower than $1.5 \mathrm{GHz}$ band, which is around $1.45 \mathrm{GHz}$.

The impedance matching in the target frequencies frequency range needs to be achieved for realizing the phase shifter. In the periodic transmission line structures, the Bloch impedance is obtained from

$$
Z_{B}=j \frac{2 \omega L_{L} \kappa}{\left(\omega^{2} C_{R} L_{L}-1\right)\left(\kappa-\sqrt{\kappa^{2}-4 \omega^{2} C_{L} L_{L} \kappa}\right)},
$$

where

$$
\kappa=\left(\omega^{2} C_{L} L_{R}-1\right)\left(\omega^{2} C_{R} L_{L}-1\right) .
$$

To achieve voltage standing wave ratio (VSWR) is less than 2 in the considering frequenciesfrequency range, the third condition is $40<Z_{B}<60[\Omega]$.

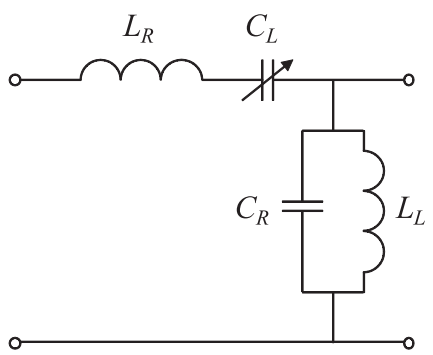

Fig. 5. Equivalent circuit model for the ideal CRLH transmission line.

The phase constant is derived from

$$
\begin{aligned}
\beta & =\frac{1}{p} \cos ^{-1}\left(1+\frac{1}{2}\left(j \omega L_{R}+\frac{1}{j \omega C_{L}}\right)\left(\frac{1}{j \omega L_{L}}+j \omega C_{R}\right)\right) \\
& =\frac{1}{p} \cos ^{-1}\left(1-\frac{1}{2}\left(\frac{1}{\omega^{2} L_{L} C_{L}}+\omega^{2} L_{R} C_{R}-\frac{L_{R}}{L_{L}}-\frac{C_{R}}{C_{L}}\right)\right),
\end{aligned}
$$

where $p$ is periodic length of the unit cell. To achieve the guided wave propagation in the target frequency bands, the phase constant in the considering frequency bands should be in the slow wave region.

We estimate the phase shift that can be obtained by a unit cell composed of a CRLH transmission line at 1.5 and $2 \mathrm{GHz}$, which are considered as the representative frequencies of 1.5 and $2 \mathrm{GHz}$ bands. In this investigation, the phase constants are calculated by changing $C_{L}$ in Equation (6). The reason of changing $C_{L}$ is that $C_{L}$ is largely influenced on the phase characteristic and it is relatively easy to change the value compared with other three parameters. The detail of the calculation is as follows.

We first determine the range of the circuit parameters satisfying the above first condition $\left(f_{\mathrm{sh}}\right.$ and $f_{\mathrm{se}}>2 \mathrm{GHz}$ here). As the results of our preliminary investigation, the right-handed circuit parameters $L_{R}$ and $C_{R}$ are set to $4.5 \mathrm{nH}$ and $0.2 \mathrm{pF}$, respectively, in this study. These values come from the final CRLH transmission line structure. The variable ranges of the left-handed circuit parameters satisfying the first condition are $1 \mathrm{nH}<L_{L}<31 \mathrm{nH}$ and $0.1 \mathrm{pF}<C_{L}<1.4 \mathrm{pF}$. The phase constants are calculated by using the four parameters with varying $L_{L}$ and $C_{L}$. The phase shift is determined by the difference of the phase constant between a considering $C_{L}$ and $C_{L}$ of $0.1 \mathrm{pF}$ when $L_{L}$ is fixed. We also define the phase shift difference (PSD). This is the key parameter of the proposed phase shifter. The PSD is the difference between the phase shift at $1.5 \mathrm{GHz}$ and that at $2 \mathrm{GHz}$ when the same four circuit parameters are used. Figure 6 shows the PSD between 1.5 and $2 \mathrm{GHz}$ depending on $C_{L}$ and $L_{L}$. The black area in the figure shows the area that the PSD is not available. As can be seen in Figure 6, the variable range of $C_{L}$ obtaining large PSD variation can be reduced when increasing $L_{L}$ from $1 \mathrm{nH}$ to around $13 \mathrm{nH}$. As increasing $L_{L}$ from $13 \mathrm{nH}$, the largest PSD at the fixed $L_{L}$ increases and the largest PSD is maximum when $L_{L}$ is $19 \mathrm{nH}$. The maximum PSD is $66^{\circ}$ at 


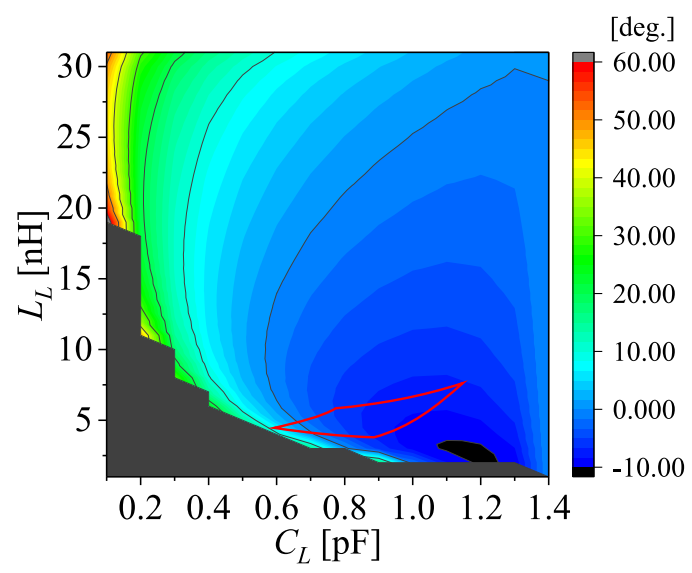

Fig. 6. Phase shift difference between 1.5 and $2 \mathrm{GHz}$ depending on $C_{L}$ and $L_{L}$.

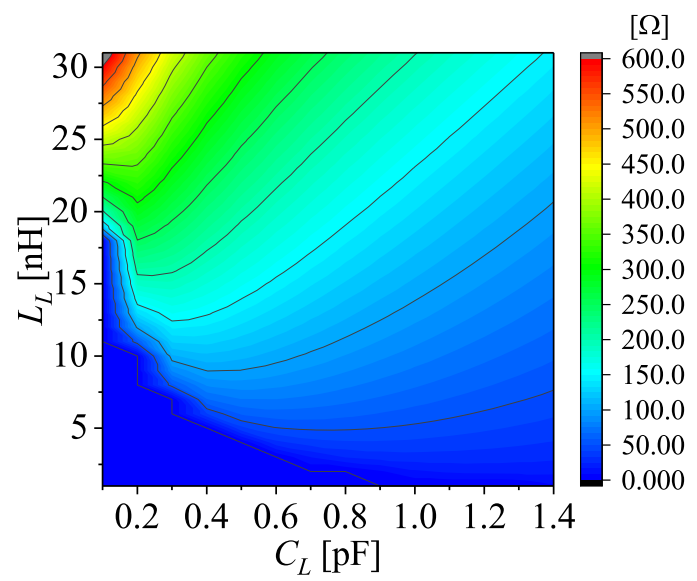

Fig. 7. Bloch impedance $Z_{B}$ at $1.5 \mathrm{GHz}$ depending on $C_{L}$ and $L_{L}$.

$C_{L}$ of $0.1 \mathrm{pF}$. When further increasing $L_{L}$ from $19 \mathrm{nH}$, the largest PSD at the fixed $L_{L}$ gradually decreases and the variable range of $C_{L}$ obtaining large PSD variation increases.

Figures 7 and 8 show the Bloch impedance $Z_{B}$ at 1.5 and $2 \mathrm{GHz}$ depending on $C_{L}$ and $L_{L}$. The area satisfying $40<Z_{B}<60$ at both $1.5 \mathrm{GHz}$ and $2 \mathrm{GHz}$ is plotted by red closed line in Figure 6. This corresponds to the second condition. When considering the second condition, the largest PSD variation can be obtained when $L_{L}$ is around 4 to $5 \mathrm{nH}$ and $C_{L}$ is from 0.5 to $0.9 \mathrm{pF}$. The estimated PSD variation is around $10^{\circ}$ to $15^{\circ}$.

\section{Unit cell design}

This section investigates an actual CRLH transmission line configuration. Microstrip line based CRLH transmission line is considered and mushroom configuration is applied to configure the unit cell of the CRLH transmission line. Figure 9 shows the unit cell structure of the considering CRLH transmission line. Shunt inductor $L_{L}$ is realized by

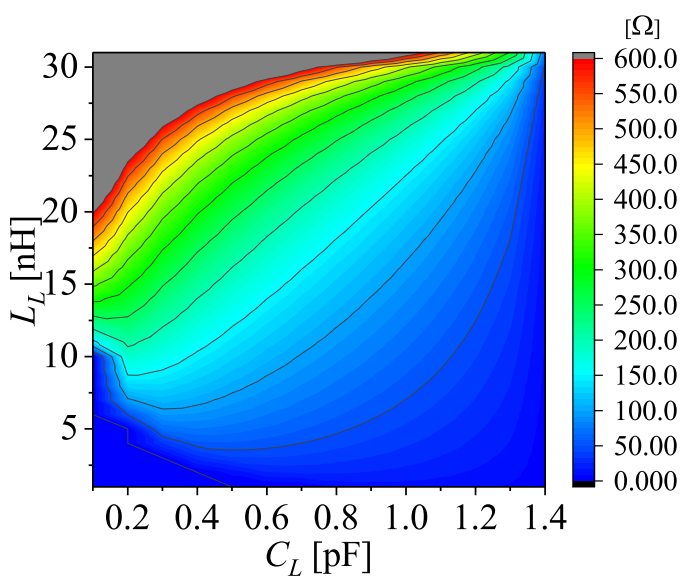

Fig. 8. Bloch impedance $Z_{B}$ at $2 \mathrm{GHz}$ depending on $C_{L}$ and $L_{L}$.

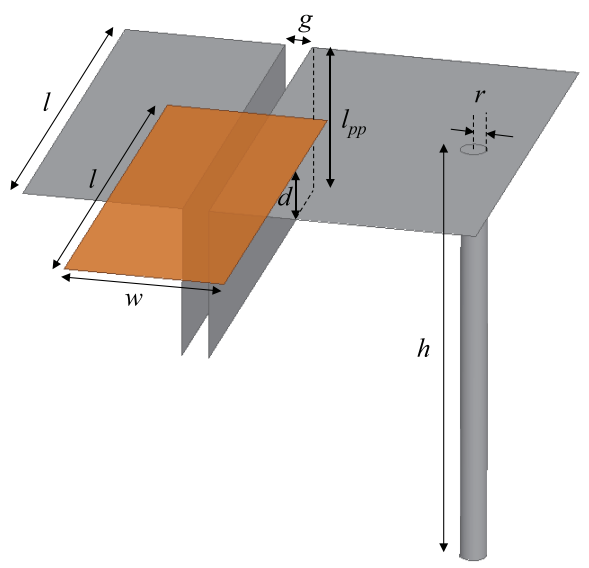

Fig. 9. Configuration of unit cell structure.

the shorted pin and series capacitor $C_{L}$ is realized by the parallel plate capacitor. MIM capacitors are added by arranging the additional patches above the parallel plate capacitors. The phase characteristics are varied by sliding the patch mechanically in $x$ direction. The crossover between the additional patch and the main patch is $50 \%$ as shown in Figure 9. The crossover of $100 \%$ corresponds to the additional patch is located directly above the main patch. The sliding range of the proposed phase shifter is defined as the range from crossover of $10 \%$ to $100 \%$.

In this section, the influence of structural parameters on the PSD variation is investigated. We first investigate the influence of the height $h$ and the radius $r$ on the PSD variation. Those parameters mainly correspond to the shunt inductance $L_{L}$. Figure 10 shows the PSD variation between the crossover of $10 \%$ and $100 \%$ depending on the height $h$ and radius $r$ of the shorted pin. The finite element method is used for the electromagnetic simulation. $l_{p p}=$ $2.9 \mathrm{~mm}$ and $d=0.30 \mathrm{~mm}$ in this simulation. The PSD variation is maximum when the height $h$ is $6 \mathrm{~mm}$ and the radius $r$ is $0.25 \mathrm{~mm}$. The second condition (matching condition) is to satisfy the Bloch impedance in between 40 


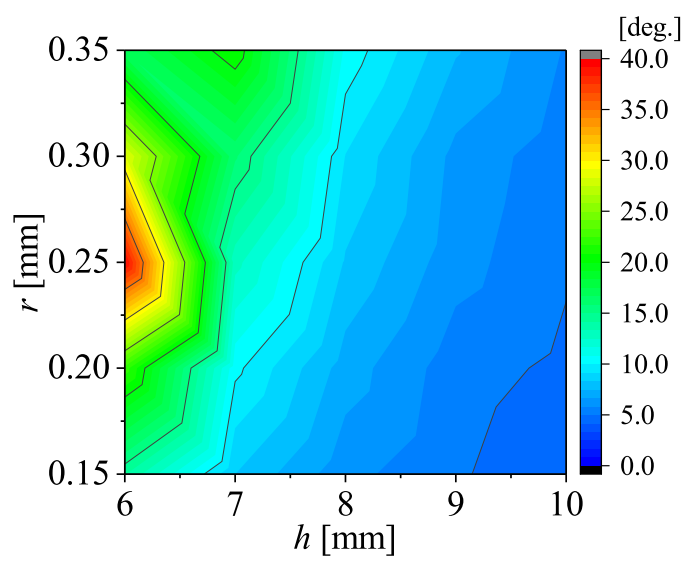

Fig. 10. Phase shift difference by subtracting phase shift between crossover of $10 \%$ and $100 \%$ at $2 \mathrm{GHz}$ from that at $1.5 \mathrm{GHz}$ when $h$ and $r$ are varied.

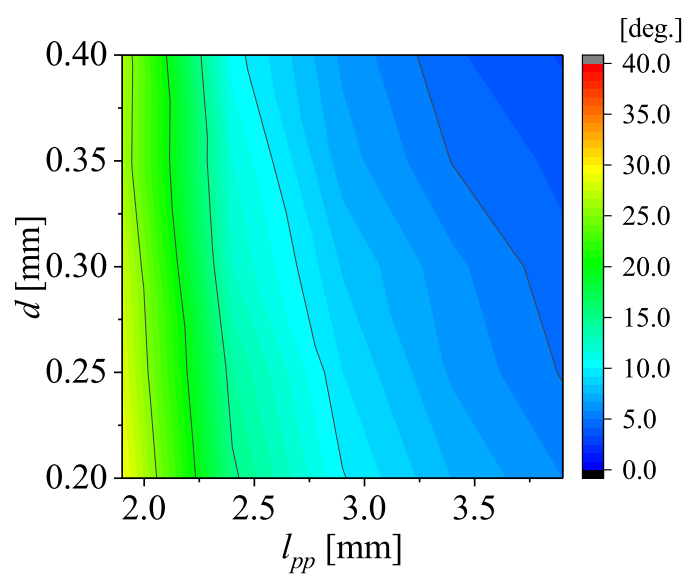

Fig. 11. Phase shift difference by subtracting phase shift between crossover of $10 \%$ and $100 \%$ at $2 \mathrm{GHz}$ from that at $1.5 \mathrm{GHz}$ when $l_{p p}$ and $d$ are varied

and $60 \Omega$, which satisfies the VSWR of less than 2 for $50 \Omega$. The area with the height $h$ of around $8 \mathrm{~mm}$ and the radius $r$ of $0.25 \mathrm{~mm}$ satisfies this condition and the PSD variation of about $10^{\circ}$ is achieved in the area. The $L_{L}$ of the shorted pin is estimated from the microstrip line with shorted pin model. In other word, the parallel plate capacitor and the additional patch are removed from the unit cell model. $Z$ parameters of the model are calculated and the $L_{L}$ is derived by applying T-shape two-port network to the model. The estimated $L_{L}$ at the height $h$ of 6,8 , and $10 \mathrm{~mm}$ are $3.6,5.0$, and $6.5 \mathrm{nH}$, respectively, when the radius $r$ is $0.25 \mathrm{~mm}$. The derived $L_{L}$ values agree with $L_{L}$ estimated from the equivalent circuit.

Figure 11 shows the PSD variation between the crossover of $10 \%$ and $100 \%$ when the height of the parallel plate capacitor $l_{p p}$ and the thickness of the insulator of the MIM capacitor $d$ are varied. These correspond to the series capacitance $C_{L}$. The height $h$ is $8 \mathrm{~mm}$ and the radius $r$ is $0.25 \mathrm{~mm}$. The PSD variation increases as decreasing $l_{p p}$.

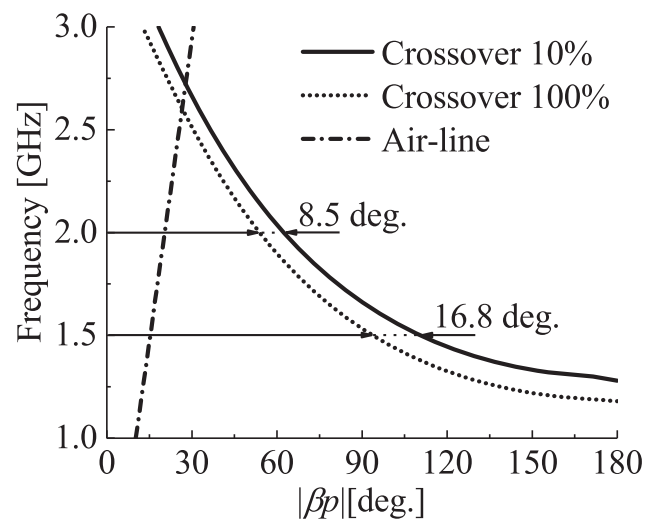

Fig. 12. Dispersion diagram of the unit cell with $l=8 \mathrm{~mm}$, $g=0.5 \mathrm{~mm}, p=8.5 \mathrm{~mm}, w=3 \mathrm{~mm}, r=0.25 \mathrm{~mm}, h=8 \mathrm{~mm}, l_{p p}=$ $2.9 \mathrm{~mm}, d=0.3 \mathrm{~mm}$.

The thickness $d$ is not sensitive to the PSD variation. To satisfy second condition for the Bloch impedance, the values around $l_{p p}$ of $2.9 \mathrm{~mm}$ and $d$ of $0.30 \mathrm{~mm}$ are suitable in this configuration. The $C_{L}$ of the parallel plate capacitor with the additional patch is also estimated from the model removing the shorted pin from the unit cell model. The estimated $C_{L}$ at the height $l_{p p}$ of $1.9,2.9$, and $3.9 \mathrm{~mm}$ are $0.65,0.93$, and $1.27 \mathrm{pF}$ for crossover of $10 \%$ and $0.89,1.21$, and $1.65 \mathrm{pF}$ for crossover of $100 \%$ respectively when the thickness $d$ is $0.3 \mathrm{~mm}$. Although the values are a little larger than the values obtaining the PSD variation of around $10^{\circ}$ to $15^{\circ}$ shown in Figure 6, the values fairly agree with those shown in Figure 6. Therefore, it can be said that the designed mushroom configuration can satisfy the electrical requirements for the proposed phase shifter described in Section 3.

Figure 12 shows the dispersion diagram of the unit cell after parametric study. In the proposed phase shifter, the phase difference at $1.5 \mathrm{GHz}$ and $2 \mathrm{GHz}$ are $16.8^{\circ}$ and $8.5^{\circ}$. Thus, the phase difference at $1.5 \mathrm{GHz}$ is larger than that at $2 \mathrm{GHz}$. This is because the unit cell is working in the lefthanded region. This dispersion relation cannot be achieved in the conventional transmission line. Since the final tilt angle can be achieved by the summation of the phase shifts of the conventional and proposed phase shifters, the phase shift by using the conventional phase shifter has to be designed again according to that by using the proposed phase shifter.

\section{Experiments}

The proposed CRLH transmission line is experimentally investigated. As described in Section 2, the required PSD variation is around $30^{\circ}$ to $50^{\circ}$ for a typical base station antenna. Since the unit cell of the considering structure has around $10^{\circ} \mathrm{PSD}$ variation, cascade structure should be required for the phase shifter. In this study, 5-cell structure is fabricated, which corresponds to equalize the directivity of a base station antenna between 1.5 and $2 \mathrm{GHz}$ toward the vertical angle of about $1^{\circ}$. 


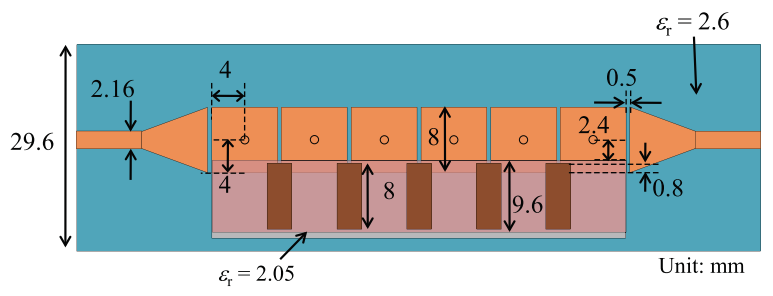

(a)

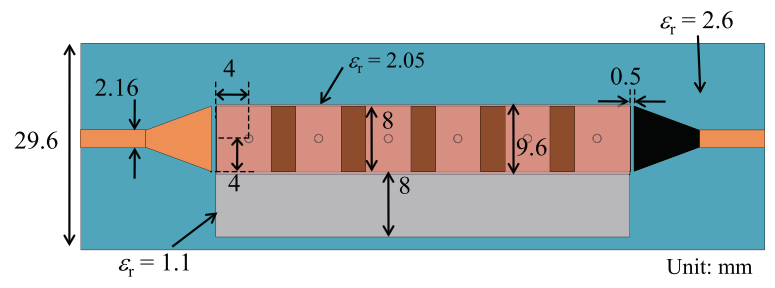

(b)

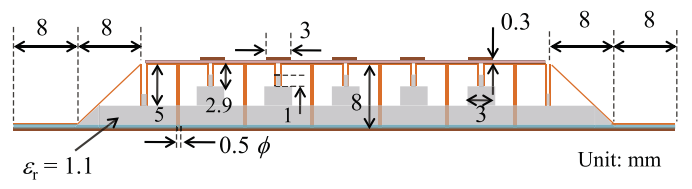

(c)

Fig. 13. Configuration of fabricated phase shifter composed of 5-cell CRLH transmission line. (a) Top view of crossover of $10 \%$. (b) Top view of crossover of $100 \%$. (c) Side view.

Figure 13 shows the configuration of the fabricated phase shifter composed of the 5-cell CRLH transmission line with input and output circuits. The formed materials with $\varepsilon_{r}=1.1$ are employed for supporting each parallel plate capacitors of the CRLH transmission line and the slide region of the MIM capacitors. The simulated PSD of the unit cell used in this configuration is $10.1^{\circ}$. To cancel out the inductive reactance of the 5-cell structure without the strip and tapered lines at the input and output sides in Figure 13, the parallel plate capacitors with $l_{p p}$ of $5 \mathrm{~mm}$ are inserted at the input and output sides of the CRLH transmission line. $50 \Omega$ microstrip lines on the dielectric substrate with $8 \mathrm{~mm}$ length and $0.8 \mathrm{~mm}$ thickness are connected to both the input and output sides. The taper structures with $8 \mathrm{~mm}$ length are connected from microstrip line to the CRLH transmission line. The phase shifter is packaged by the blass case. The inner conductors of the coaxial cables are connected to the input and output microstrip lines.

Figure 14 shows the magnitude of $S$-parameter characteristics of the fabricated phase shifter. The simulated result is also plotted in Figure 14. The measured results well agree with the simulated results. The measured $\left|S_{11}\right|$ and $\left|S_{21}\right|$ of the fabricated phase shifter with the crossover of $100 \%$ become $-6.7 \mathrm{~dB}$ and $-2.0 \mathrm{~dB}$ at $1.5 \mathrm{GHz}$. Figure 15 shows the phase of $S_{21}$ characteristics of the fabricated phase shifter and simulated one. Here, the phase shift is determined by the difference of the phases of $S_{21}$ between the crossover of $10 \%$ and $100 \%$ and the PSD is the

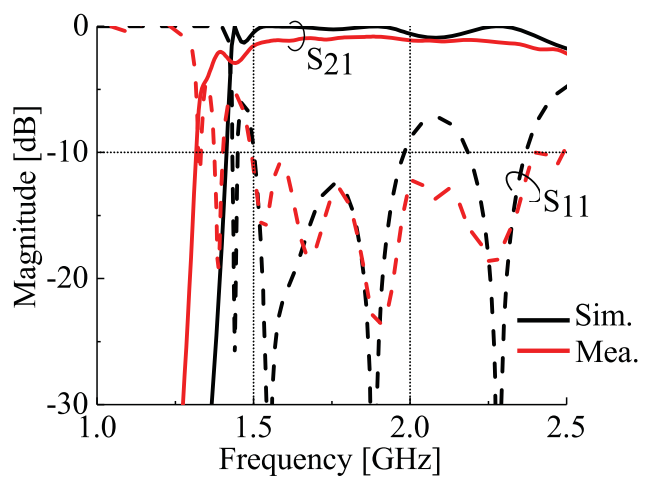

(a)

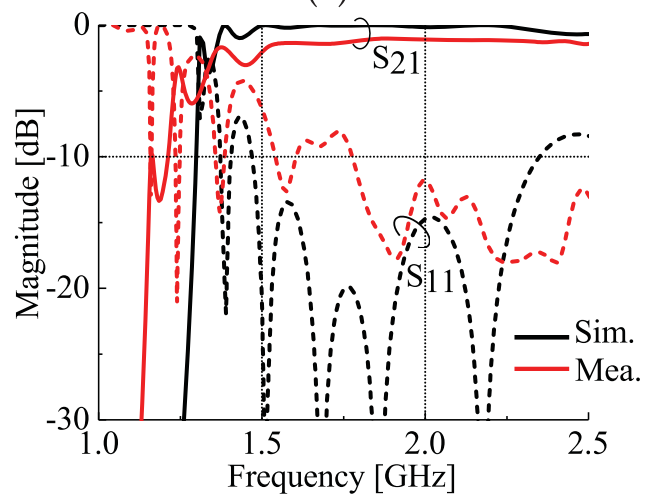

(b)

Fig. 14. Magnitude of S-parameters characteristics of 5-cell transmission line. (a) Crossover of $10 \%$. (b) Crossover of $100 \%$.

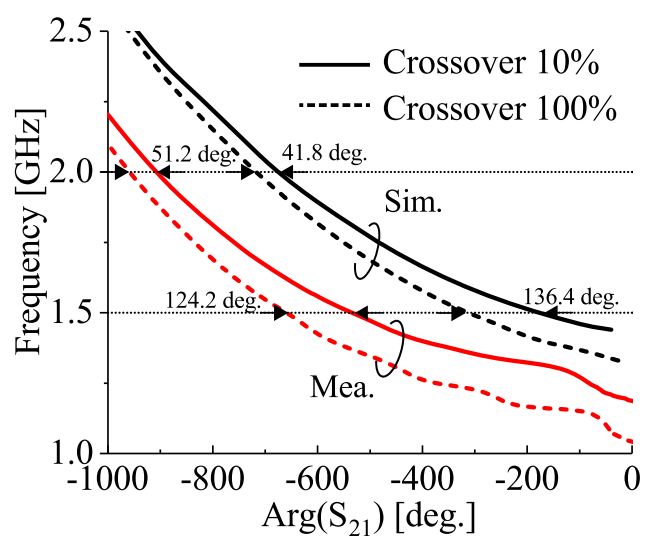

Fig. 15. Phase of $S_{21}$ characteristics of 5-cell transmission line.

difference between the phase shift at $1.5 \mathrm{GHz}$ and that at $2 \mathrm{GHz}$ of the 5-cell CRLH transmission line. The simulated phase shifts at $1.5 \mathrm{GHz}$ and $2 \mathrm{GHz}$ are $136.4^{\circ}$ and $41.8^{\circ}$, and the measured phase shifts at $1.5 \mathrm{GHz}$ and $2 \mathrm{GHz}$ are $124.2^{\circ}$ and $51.2^{\circ}$, respectively. The PSD of the 5-cell CRLH transmission line is $94.6^{\circ}$ in the simulation and $73.0^{\circ}$ in the measurement. These values correspond to about $18.9^{\circ}$ and $14.6^{\circ}$ for the unit cell, respectively. Fabrication error should be a reason of the discrepancies between simulated and 
measured results. However, the measured results fairly agree with the simulated results and the large phase shift at lower frequency, which is required for the proposed phase shifter is verified through experiment. The measured PSD per unit cell of the 5-cell structure is $6.3^{\circ}$ larger than that of the unit cell described in Section 4. This should be due to the mutual coupling between the adjacent cells.

\section{Conclusion}

This paper presents a new phase shifter for multi-band mobile base station antennas that can make deeper tilt in low frequency by using CRLH transmission line. The unit cell of the proposed CRLH transmission line is composed of a mushroom type structure. The series capacitance is realized by folding the top plates of the mushrooms along the transmission line. In addition, additional patches are placed above the gap between the adjacent unit cells. The additional patches can be mechanically shifted, then the phase characteristics can be varied.

To design the unit cell of the CRLH transmission line, circuit parameters that satisfy the electrical requirements are first investigated by using T shape 2-port network. Then, the actual structure of the unit cell satisfying the derived circuit parameters is investigated by the finite element method analysis. Finally, a 5-cell CRLH transmission line composed of the designed unit cells is fabricated and the inverse phase shift at two frequencies by using dispersion relation of CRLH transmission line is verified by the simulation and measurement. Large phase shift at lower frequency provides the reduction of the horizontal directivity in the base station antenna. As a result, the interference to neighboring cell at lower frequency can be suppressed. Realizing a phase shifter combining the proposed CRLH transmission line with conventional phase shifter, and miniaturization of the CRLH transmission line are future work.

This work was supported by JSPS KAKENHI Grant Number 16K06365 and The Telecommunications Advancement Foundation.

\section{References}

1. W.C.Y. Lee, Mobile communications engineering (McGrawHilee, 1982), pp. 5-9

2. Y. Yamada, Y. Ebine, K. Tsunekawa, Base and mobile station antennas for land mobile radio systems, IEICE Trans. Commun. E74, 1547 (1991)
3. M. Kijima, Y. Ebine, Y. Yamada, Development of a dualfrequency base station antenna for cellular mobile radios, IEICE Trans. Commun. E82-B, 636 (1999)

4. H. Arai, K. Cho, Cellular and PHS base station antenna systems, IEICE Trans. Commun. E86-B, 980 (2003)

5. N. Honma, F. Kira, T. Maruyama, K. Cho, H. Mizuno, Offset beam planar antenna employing low loss triangular dielectric phase shifter, IEICE Trans. Commun. E86-B, 2720 (2003)

6. R.B. Hwang, Y. Chang, M.-I. Lai, A low-cost electrical beam tilting base station antennas for wireless communication system, IEEE Trans. Antennas Propag. 52, 115 (2004)

7. C. Caloz, T. Itoh, Electromagnetic metamaterials: Transmission line theory and microwave applications (Wiley-IEEE Press, 2005)

8. M.A. Antoniades, G.V. Eleftheriades, Compact linear lead/ lag metamaterial phase shifters for broadband applications, IEEE Antennas Wirel. Propag. Lett. 2, 103 (2003)

9. H. Kim, A.B. Kozyrev, A. Karbassi, D.W. van der Weide, Linear tunable phase shifter using a left-handed transmission line, IEEE Microw. Wirel. Compon. Lett. 15, 366 (2005)

10. C. Damm, M. Schussler, M. Oertel, R. Jakoby, Compact tunable periodically LC loaded microstrip line for phase shifting applications, in International Microwave Symposium Digest. IEEE, 2003 (2005), pp. 2003-2006

11. P. He, P.V. Parimi, Y. He, V.G. Harris, C. Vittoria, Tunable negative refractive index metamaterial phase shifter, Electron. Lett. 43, 1440 (2007)

12. A. Grbic, G.V. Eleftheriades, A backwad-wave antenna based on negative refractive index L-C networks, IEEE Antennas and Propagation Society International Symposium. IEEE, 340 (2002) pp. 340-343

13. L. Liu, C. Caloz, T. Itoh, Dominant mode leaky-wave antenna with backfire-to-endfire scanning capability, Electron. Lett. 38, 1414 (2002)

14. N. Michishita, H. Kitahara, Y. Yamada, K. Cho, Tunable phase shifter using composite right/left-handed transmission line with mechanically variable MIM capacitors, IEEE Antennas Wirel. Propag. Lett. 10, 1579 (2011)

15. N. Michishita, Y. Yamada, K. Cho, Achievement of inverse frequency-dependent phase shift by using composite right/ left-handed phase shifter, Proc. Int. Symp. Antennas Propag. 4E1-4, 1510 (2012)

16. Y. Inoue, K. Cho, MIMO base station antenna employing mode selection in vertically split array, in 5th European Conference on Antennas Propagation (2011), pp. 2751-2755

17. S. Asaka, M. Karikomi, Y. Ebine, The structure and characteristics of a revolving variable phase shifter using slot couplings, Proc. Int. Symp. Antennas Propag. 1E2-3, $305(2000)$ 